\title{
PERCEPÇÃO DOS CONSUMIDORES DE SOJA E DERIVADOS NA CIDADE DE JÚLIO DE CASTILHOS (RS) - BRASIL
}

\author{
Dalva Maria Righi Dotto* \\ Rafaela Rosa Colpo** \\ Silvia Cristina Ferreira Iop ${ }^{* * *}$ \\ Andreia Cirolini****
}

RESUMO: O Brasil ocupa o segundo lugar no ranking mundial de produção de soja desde 2003, sendo que o complexo derivado deste grão tem expressiva contribuição econômica no país. O movimento dos consumidores no intuito de ter uma alimentação mais saudável quanto à qualidade e aspectos nutricionais dos alimentos impulsionou a indústria para o desenvolvimento de uma maior variedade de produtos e, neste contexto, a soja tem se mostrado como uma importante fonte nutritiva. Este estudo objetivou avaliar a percepção dos consumidores em relação a alimentos derivados da soja. Para atingir os objetivos propostos foi realizada uma pesquisa descritiva sobre o conhecimento e aceitação dos alimentos derivados, sendo que os resultados indicaram que a maior parte da amostra já consumiu estes alimentos, entretanto, os que consomem atualmente formam um grupo menor de consumidores. Também se constatou desconhecimento das propriedades nutricionais e funcionais do grão para a saúde humana pela maior parte dos entrevistados, sendo esta uma das prováveis causas do baixo consumo e da falta de interesse da população pela introdução de alimentos derivados da soja nos cardápios diários.

PALAVRAS-CHAVE: Agronegócio; Ciência de Alimentos; Mercado Consumidor; Soja.

\section{PERCEPTION OF CONSUMERS FOR SOYBEANS AND ITS DERIVATIVES IN JÚLIO DE CASTILHOS, BRAZIL}

ABSTRACT: Since 2003, Brazil ranks second in soybean production and the derivatives constitute an expressive economic contribution for the country.

Doutora em Desenvolvimento Regional pela Universidade de Santa Cruz do Sul; Docente Adjunta da Universidade Federal de Santa Maria - UFSM, Brasil; E-mail: dalvadotto@gmail.com

** Tecnóloga em Agronegócio; Universidade Federal de Santa Maria - UFSM, Brasil.

*** Doutora em Ciências dos Alimentos pela Universidade Federal de Santa Catarina - UFSC, Brasil; Docente Adjunta da Universidade Federal de Santa Maria - UFSM, Brasil.

**** Doutora em Ciências dos Alimentos pela Universidade Federal de Santa Catarina - UFSC, Brasil; Docente Adjunta da Universidade Federal de Santa Maria - UFSM, Brasil. 
Consumers' movements for healthier food in quality and nutrition aspects triggered the industries to develop a greater variety of products. Soybean is actually a high nutrition food source. Current analysis evaluates the perception of consumers on soybean derivatives and aims at an in-depth knowledge and acceptance of derived food. Results reveal that most people in the sample have already consumed the food even though current group is formed by a lower number of consumers. The nutritional and functional properties of soybeans for human health are not known by most interviewed people and this fact may have been the cause of low consumption and lack of interest of the population in the introduction of soybean derivatives in daily menus.

KEY WORDS: Agribusiness; Food Science; Consumer Market; Soybean.

\section{INTRODUÇÃO}

A soja se tornou uma das commodities mais relevantes na economia brasileira. Sendo o segundo produtor mundial do grão, o Brasil, segundo Vencato et al. (2010), projeta um cenário positivo com aumento de $40 \%$ até 2020 , enquanto que nos Estados Unidos, atualmente dominante na produção, o crescimento no mesmo período deverá ser no máximo de 15\%. Com essa projeção, o Brasil atingirá mais de 105 milhões de toneladas, tornando-se o maior produtor (AMAZONAS, 2013).

A cultura da soja ocupa um papel fundamental no agronegócio da América do Sul, onde o Brasil, a Argentina e o Paraguai respondem por $50 \%$ da produção mundial (DEMARCHI, 2011).

Para Freitas (2011), os principais fatores do avanço da soja foram: mecanização e criação de cultivares altamente produtivas adaptadas às diversas regiões, desenvolvimento de pacotes tecnológicos relacionados ao manejo (de solos, adubação e calagem, pragas e doenças) e identificação e solução para os principais fatores responsáveis por perdas no processo de colheita.

A introdução da soja no Brasil ocorreu no início do século XX e o primeiro registro de cultivo foi no município de Santa Rosa, no Rio Grande do Sul, em virtude das semelhanças climáticas do ecossistema de origem (Sul dos Estados Unidos) (EMBRAPA, 2004). 
Segundo Lazzarotto e Hirakuri (2010), nos Estados da região Sul predomina a produção em áreas inferiores a 100 hectares, podendo ser caracterizada a grande maioria como agricultores familiares. Nas demais regiões, a produção tende a ser explorada em áreas superiores a 500 hectares, caracterizando-se como agricultores patronais.

O Rio Grande do Sul foi o maior produtor nacional de soja entre a década de 60 até meados de 90. De 1996 a 1999 o Paraná assumiu esta posição e, desde o início dos anos 2000, o Mato Grosso lidera o ranking (DEMARCHI, 2011), seguido pelo Paraná e Rio Grande do Sul totalizando, nestes três Estados, mais de 50\% da produção nacional (IBGE, 2012) (Tabela 1).

Tabela 1. Produção de soja em grão por Estado brasileiro - safras de 2008/09 a 2012/13 em milhões de toneladas

\begin{tabular}{lccccc}
\hline \multicolumn{1}{c}{ Estados } & \multicolumn{5}{c}{ Safras } \\
\cline { 2 - 6 } & $\mathbf{2 0 0 8 / 0 9}$ & $\mathbf{2 0 0 9 / 1 0}$ & $\mathbf{2 0 1 0 / 1 1}$ & $\mathbf{2 0 1 1 / 1 2}$ & $\mathbf{2 0 1 2 / 1 3}\left(^{*}\right)$ \\
\hline Mato Grosso & 17,96 & 18,77 & 20,41 & 21,85 & 23,70 \\
\hline Paraná & 9,51 & 14,08 & 15,42 & 10,94 & 15,08 \\
Rio Grande do Sul & 7,91 & 10,22 & 11,62 & 6,53 & 11,96 \\
\hline Goiás & 6,84 & 7,34 & 8,18 & 8,25 & 8,79 \\
\hline Mato Grosso do Sul & 4,18 & 5,31 & 5,17 & 4,63 & 6,17 \\
\hline Bahia & 2,42 & 3,11 & 3,51 & 3,18 & 3,76 \\
\hline Minas Gerais & 2,75 & 2,87 & 2,91 & 3,06 & 3,25 \\
\hline Maranhão & 0,98 & 1,33 & 1,60 & 1,65 & 1,73 \\
\hline São Paulo & 1,31 & 1,59 & 1,71 & 1,60 & 1,72 \\
Santa Catarina & 0,97 & 1,35 & 1,49 & 1,09 & 1,53 \\
\hline Piauí & 0,77 & 0,87 & 1,14 & 1,26 & 1,40 \\
Tocantins & 0,86 & 1,07 & 1,23 & 1,38 & 1,38 \\
Outros & 0,72 & 0,79 & 0,92 & 0,96 & 0,97 \\
\hline
\end{tabular}

Fonte: (MOREIRA, 2013)

(*) Estimativa

A agricultura do Rio Grande do Sul (FONTANA et al., 2001) se caracteriza por culturas primavera-verão, correspondendo a mais de $90 \%$ da produção total de grãos. Soja e milho são as culturas de maior expressão e representam $70 \%$ da área cultivada e mais de $60 \%$ da produção total de grãos. A sobrevivência da agricultura 
familiar da soja ocorre devido à possibilidade de produção em rotação com o trigo, diferentemente das demais regióes do país, e a maior proximidade dos portos de importação de insumos e exportação da produção, reduzindo os custos gerais de transporte (SCHLESINGER, 2008).

Embora com saldo positivo, conforme Lazzarotto e Hirakuri (2010), os preços pagos e recebidos são fontes de grandes incertezas, pois são influenciados por inúmeras variáveis, principalmente econômicas e agroecológicas (fertilizantes, sementes, herbicidas, fungicidas, maquinários e mão de obra).

O processamento industrial da soja no Brasil teve início na década de 1950, sendo realizado por empresas nacionais de pequeno porte. A primeira unidade industrial foi a Incobrasa, no Rio Grande do Sul. Com grande expansão na década de 1970 e início de 80, devido às políticas governamentais que apoiaram as agroindústrias, atualmente a industrialização se diferencia pela capacidade instalada de esmagamento e pela origem de seu capital, podendo ser nacionais ou estrangeiras, dividindo-se em esmagadoras, integradas e comercializadoras (SANTOS; BACHA, 2003).

Com o crescimento dos setores envolvidos com a soja por meio de investimentos em tecnologias, novas áreas agrícolas e indústrias de processamento de grãos e refino de óleos têm se instalado com resultados positivos e avanços no território brasileiro (Tabela 2).

Tabela 2. Panorama do complexo da soja em grãos, farelo e óleo no Brasil - safra 2005/06 e 2012/13 em milhões de toneladas

\begin{tabular}{lcccccc}
\hline \multirow{2}{*}{ Safra } & \multicolumn{2}{c}{ Grão } & \multicolumn{2}{c}{ Farelo } & \multicolumn{2}{c}{ Óleo } \\
\cline { 2 - 7 } & $\mathbf{2 0 0 5 / 0 6}$ & $\mathbf{2 0 1 2 / 1 3 *}$ & $\mathbf{2 0 0 5 / 0 6}$ & $\mathbf{2 0 1 2 / 1 3}$ & $\mathbf{2 0 0 5 / 0 6}$ & $\mathbf{2 0 1 2 / 1 3}$ \\
\hline Estoque inicial & $2.734,7$ & 395,5 & $1.824,6$ & $1.598,5$ & 279,0 & 100,5 \\
Produção & $55.027,1$ & $81.510,5$ & $21.918,0$ & $29.452,5$ & $5.479,5$ & $7.458,8$ \\
Importação & 48,8 & 50,0 & 152,4 & 36,0 & 25,4 & 1,0 \\
Suprimento & $57.810,6$ & $81.956,0$ & $23.895,0$ & $31.087,0$ & $5.783,9$ & $7.560,3$ \\
Esmagamento & $28.100,0$ & $38.250,0$ & - & - & - & - \\
Semente e outros & $2.283,0$ & $3.778,7$ & - & - & - & - \\
Consumo & $30.383,0$ & $42.028,7$ & $9.780,0$ & $14.325,0$ & $3.150,0$ & $5.640,0$ \\
Interno & $24.957,9$ & $36.410,0$ & $12.332,4$ & $14.925,0$ & $2.419,4$ & $1.750,0$ \\
Exportação & & & & & & \\
\hline
\end{tabular}




\begin{tabular}{lllllll} 
Estoque final & $2.469,7$ & $3.517,3$ & $1.782,6$ & $1.837,0$ & 214,5 & 170,3 \\
\hline
\end{tabular}

Fonte: (AMAZONAS, 2013)

(*) Estimativa

No ano de 2011 foram movimentados cerca de US\$24 bilhões apenas nas exportações de soja em grãos, farelo e óleo e gerados 1,5 milhões de empregos em 17 Estados do Brasil (ABIOVE, 2012). Segundo Demarchi (2011) estes produtos lideram as exportações e, nos últimos três anos, aumentaram e geraram, em média, receita de US\$ 71 bilhões/ano.

China e União Europeia continuam sendo os grandes importadores de soja em grão. Os chineses tornaram-se os principais consumidores respondendo por $59,8 \%$ das exportações, quase cinco vezes a mais do que a União Europeia (DEMARCHI, 2011).

Três quartos da produção são destinados ao mercado externo e a China importa o grão (66\%) e farelo de soja (60\%). O óleo segue para Argélia (6\%), Irã (5\%), Índia (5\%), Cuba (4\%), entre outros (SCHLESINGER, 2008).

A indústria nacional transforma, por ano, cerca de 30,7 milhões de toneladas de soja, produzindo 5,8 milhões de toneladas de óleo comestível e 23,5 milhões de toneladas de farelo proteico, contribuindo para a competitividade nacional na produção de carnes, ovos e leite. A soja e o farelo brasileiros possuem alto teor de proteína e padrão de qualidade Premium, o que permite sua entrada em mercados extremamente exigentes como União Europeia e Japão (MAPA, 2013).

De toda a produção de biodiesel, que já alcança quase 2,5 milhões de litros ao ano no Brasil (ANP, 2013), é estimado que o óleo de soja representa mais de $80 \%$ da matéria-prima utilizada, seguido por gordura bovina $(12,4 \%)$ e óleo de algodão (2,1\%).

Tanto na alimentação animal, através de farelos e rações, quanto na produção do biodiesel e na alimentação humana, a soja se tornou uma das matériasprimas mais utilizadas pela indústria em óleo, sucos, sorvetes, biscoitos, temperos, entre outros. Por possuir destacada qualidade nutritiva, os alimentos derivados de soja estão em consonância com o crescente interesse do mercado consumidor de produtos que proporcionam benefícios relacionados à saúde.

Com a expectativa de aumentar o interesse da população por alimentos 
mais saudáveis e a constatação de que derivados da soja atendem a essa necessidade, as indústrias estão inovando em sabores e novas formas de apresentação. Neste contexto torna-se necessário o conhecimento do que o consumidor busca ao adquirir estes alimentos, pois o propósito das relações de mercado centra-se em atender e satisfazer as necessidades e desejos do usuário. Criar valor para o cliente significa a diferença entre o que o consumidor percebe quanto aos benefícios da compra e uso do produto e os custos para a sua obtenção (KOTLER; KELLER, 2006; CHURCHILL; PETER, 2010; SCHIFMANN, 2009; SOLOMON, 2011; GIGLIO, 2010).

Considerando o exposto, o estudo objetivou avaliar a percepção dos consumidores da cidade de Júlio de Castilhos (RS), Brasil, em relação a alimentos derivados da soja e, mais especificamente, identificar os fatores que impulsionam ou que impedem/limitam o consumo, o grau de conhecimento do consumidor sobre os benefícios e a frequência de uso.

\section{MATERIAL E MÉTODOS}

O procedimento metodológico adotado para alcançar os objetivos propostos nesse estudo compreende uma pesquisa descritiva de natureza quantitativa que, segundo Malhotra (2006), tem como principal objetivo a descrição de algo, normalmente de características ou funções do mercado. Como método de levantamento de informações foi realizada uma coleta estruturada de dados em 2013, na cidade de Júlio de Castilhos (RS), Brasil, que teve como base a aplicação de um questionário a 150 consumidores. A técnica de amostragem utilizada foi por conveniência, com abordagem dos consumidores nos pontos de venda de produtos derivados da soja.

A cidade de Júlio de Castilhos situa-se na região central do Rio Grande do Sul e possui 19.579 habitantes. Apresenta como principal atividade econômica os serviços, em segundo lugar a agropecuária e, em terceiro lugar, a indústria, com um PIB per capita a preços correntes em 2012 de R\$ 25.941,98. A área plantada com soja no município representa $2,61 \%$ da área territorial e a participação da soja no total da produção agrícola municipal é de 80,10\% (IBGE, 2015). Grande 
parte dos produtores rurais do município é associada da Cotrijuc - Cooperativa Agropecuária Júlio de Castilhos, que possui equipe de assistência técnica, área de comercialização de insumos, estruturas de armazenagem, produção de sementes e depósitos para fertilizantes e defensivos para auxiliar os produtores de soja do município (COTRIJUC, 2015).

Ressalta-se que, dentre os derivados da soja, foram excluídas informações relacionadas ao óleo de soja, por considerar-se que este produto já está consolidado no mercado consumidor e, portanto, não atende aos objetivos deste estudo.

\section{RESULTADOS E DISCUSSÃO}

\subsection{PERFIL DOS ENTREVISTADOS}

Do total de 150 consumidores que concordaram em participar do estudo, a contribuição de pessoas do sexo feminino foi relativamente maior (67\%). Quanto à escolaridade dos entrevistados, $69 \%$ possuíam ensino médio ou superior. A maioria (89\%) possuía renda familiar entre 1 e 5 salários mínimos ( $\mathrm{R} \$ 770,00$ a $\mathrm{R} \$ 3.850,00$ ) e estava distribuída equitativamente nas faixas etárias de 18 a 60 anos de idade.

\subsection{O CONSUMO DE ALIMENTOS DERIVADOS DA SOJA}

Embora inúmeros estudos indiquem que o consumo da soja traz benefícios para a saúde, e Chang (2001) afirme haver uma crescente utilização da soja na indústria de alimentos, constatou-se, neste estudo, que os entrevistados não possuíam o hábito de adquirir/consumir estes produtos. Quando questionados sobre o consumo dos alimentos derivados da soja apenas 34\% consumiam, $43 \%$ afirmaram já ter consumido e $23 \%$ relataram nunca ter consumido. Em relação à frequência de consumo $42 \%$ relataram consumir diariamente, $37 \%$ semanalmente, $21 \%$ mensalmente e/ou raramente. O produto derivado da soja de maior consumo foi o suco com soja (33\%) e os demais alimentos citados foram proteína texturizada de soja, iogurte e leite de soja com $11 \%$ de respostas e queijo de soja $(t o f u) \operatorname{com} 8 \%$. Outros tipos de alimentos, com pequenos índices de consumo foram: hambúrguer, 
pão, rapadura e farinha.

Os números superlativos em relação ao volume de produção da oleaginosa contrastam com a falta de hábito da população em consumir alimentos contendo soja, o que determina um mercado ainda muito incipiente no Brasil. Os produtos orientais tradicionais, como extrato solúvel (leite de soja), tofu, miso, kinako, moyashi e natto são principalmente comercializados em casas especializadas. Em supermercados os produtos de maior presença são as proteínas vegetais texturizadas, principalmente no Nordeste, como substitutos da carne, bebidas fermentadas e à base de extrato de soja, contendo ou não chocolate ou suco de frutas (CHIARELLO, 2002).

O leite de soja se constitui em uma opção de alimento para pessoas que apresentam intolerância à lactose, além de ser livre de colesterol. Apesar do grande potencial do leite de soja, o produto apresenta baixa aceitação devido ao sabor e aroma desagradáveis ao paladar dos brasileiros. Para minimizar este fator a indústria brasileira tem feito uso de novas tecnologias na obtenção do produto com melhor qualidade sensorial. A criação de novos produtos comerciais à base de extrato hidrossolúvel em combinação com sucos de frutas tem obtido êxito no mercado, indicando que os consumidores brasileiros podem estar mudando sua atitude em relação aos produtos à base de soja (CARRÃO-PANIZZI; MANDARINO, 1998; BEHRENS; SILVA, 2004).

Para facilitar a aproximação entre produtor e consumidor no processo de escolha e aquisição de produtos, foi solicitado aos entrevistados listar os motivos de consumir derivados da soja e constatou-se que os benefícios proporcionados à saúde eram o principal motivo de compra (Tabela 3).

Tabela 3. Motivos para o consumo de alimentos derivados de soja na cidade de Júlio de Castilhos (RS), Brasil, em \%

\begin{tabular}{lc}
\hline Motivos & $\%$ \\
\hline Benefícios proporcionados à saúde & 62,50 \\
Sabor agradável & 33,33 \\
Indicações médicas & 4,17 \\
\hline
\end{tabular}


De acordo com Chang (2001), os principais fatores que promoveram o interesse e a utilização crescente da soja na indústria de alimentos referem-se à sua contribuição em benefício à saúde, mudança de atitudes dos consumidores em relação à soja, melhoramento do processo tecnológico da utilização dela, crescimento da produção mundial e popularidade da dieta rica em fibras e menor teor de gordura saturada.

A soja é rica em proteínas, fitosteróis e peptídeos com baixo peso molecular, oligossacarídeos e ácidos graxos poli-insaturados, que auxiliam na redução de riscos de doenças crônicas não transmissíveis. Também constitui boa fonte de ferro, potássio, magnésio, zinco, cobre, fósforo, manganês e vitaminas do complexo B (CARRÃO-PANIZZI; MANDARINO, 1998).

Mostrando-se um alimento versátil no que se refere às possibilidades nutricionais e funcionais, a utilização da soja como substituto da proteína animal está associada aos benefícios na prevenção de problemas cardiovasculares. Os fitoestrógenos (isoflavonas ou isoflavonoides) estão sendo empregados nas terapias de reposição hormonal em mulheres na menopausa. As isoflavonas são componentes importantes no combate ao câncer por apresentarem atividade antioxidante, aumento da apoptose, inibição da angiogênese e modulação da resposta imune (YIM, 2002; KINOUCHI, 2006).

Segundo Mandarino (2002), estudos realizados no Japão e na China, cujas populações utilizam regularmente a soja em sua dieta alimentar, mostraram reduzidos índices de doenças coronárias, câncer de mama e de próstata. Além desses, a soja possui efeitos benéficos nos cânceres de bexiga, intestino, dentre outros (MESSINA et al., 1994; ADLERCREUTZ, 2002).

De acordo com a ANVISA (2013), a soja possui um teor médio de proteínas em torno de $40 \%$, enquanto o arroz $7 \%$ e feijão $20 \%$. A soja não possui amido e, em média, tem 20\% de lipídios, 5\% de minerais e 34\% de carboidratos (glicose, frutose, sacarose, fibras, rafinose e estaquiose). Há muitos milênios a soja tem sido usada nos países asiáticos como alimento e medicamento. No Ocidente, é mais conhecida pelo seu valor em proteínas e lipídios (AMARAL, 2006).

A funcionalidade da proteína de soja foi reconhecida em 1999 pelo Food and Drug Administration (FDA), que autorizou informar na rotulagem nutricional o consumo diário de 25 gramas de proteína de soja para reduzir os riscos de doenças 
do coração (PENHA et al., 2007).

No Brasil, a quantidade de proteína de soja contida na porção do produto pronto para consumo deve ser declarada no rótulo, próximo à alegação. No caso de produtos na forma de cápsulas, tabletes, comprimidos e similares, deve ser declarada a quantidade de proteína de soja na recomendação diária do produto pronto para o consumo, conforme indicação do fabricante. Os dizeres de rotulagem e o material publicitário dos produtos à base de soja não podem veicular qualquer alegação em função das isoflavonas, seja de conteúdo ('contém'), funcional, de saúde e terapêutica (prevenção, tratamento e cura de doenças) (ANVISA, 2013).

A fração oleosa do grão é rica em ácidos graxos poli-insaturados $\omega$-3 e $\omega$-6, que auxiliam na diminuição dos riscos de doenças cardiovasculares, pois reduzem os níveis de lipoproteína de baixa densidade (LDL), aumentam os de lipoproteína de alta densidade (HDL) e diminuem os de triglicerídeos (SANTOS et al., 2011).

O óleo presente nos grãos de soja em teores adequados (20\%) fornece as calorias necessárias ao organismo, permitindo que as proteínas ingeridas da dieta sejam metabolizadas para síntese de novos tecidos, ao invés de serem destinadas à produção de energia, o que é comum nas dietas de baixo conteúdo calórico (CARRÃOPANIZZI; MANDARINO, 1998). Em relação ao uso da farinha de soja e seus derivados na dieta humana, Chang (2001) analisa como uma imprescindível necessidade para manter o equilíbrio fisiológico do organismo, por promoverem a redução de risco do surgimento precoce de certas doenças crônicas não transmissíveis.

Os locais de aquisição dos alimentos derivados da soja foram, preferencialmente, os supermercados para $96 \%$ dos entrevistados, enquanto que apenas $4 \%$ compravam em lojas de produtos naturais. Na mesma proporção, 96\% afirmaram consumir estes alimentos em casa, enquanto os demais $4 \%$ em lanchonetes.

Aos entrevistados foi solicitada a escolha dos três principais motivos de não consumo de derivados da soja, sendo que $30 \%$ citaram a falta de hábito, seguido de desinteresse pessoal (23\%) e sabor desagradável (19\%) (Tabela 4). 
Tabela 4. Principais motivos que levam ao não consumo de alimentos derivados de soja na cidade de Júlio de Castilhos (RS), Brasil, em \%

\begin{tabular}{l|c}
\hline Motivos & $\%$ \\
\hline Falta de hábito & 29,71 \\
\hline Desinteresse pessoal & 23,19 \\
\hline Sabor desagradável & 18,84 \\
\hline Não encontrar (visualizar) os produtos nos pontos de venda & 16,67 \\
\hline Preço elevado & 8,70 \\
Falta de variedades & 2,17 \\
Outros & 0,72 \\
\hline
\end{tabular}

Em relação às críticas à oferta de derivados da soja, sobressai a afirmação dos entrevistados de que são poucas as informações a disposição sobre os benefícios no consumo destes alimentos. Também criticaram as poucas opções de sabores e de variedade de produtos (Tabela 5).

Tabela 5. Críticas em relação à oferta de alimentos derivados da soja na cidade de Júlio de Castilhos (RS), Brasil, em \%

\begin{tabular}{l|c}
\hline Críticas & \% \\
\hline Poucas informações sobre os alimentos & 46,58 \\
Poucas opções de sabores & 10,96 \\
Pouca variedade de produtos & 9,59 \\
Dificuldade no preparo & 1,37 \\
\hline Não possui críticas & 31,51 \\
\hline
\end{tabular}

Sobre os benefícios proporcionados à saúde através do consumo de derivados da soja, $22 \%$ dos entrevistados afirmaram que desconhecem totalmente os benefícios, $57 \%$ possuíam pouco conhecimento e $21 \%$ conheciam. Nesta perspectiva 90\% manifestaram interesse em saber mais sobre os benefícios que o consumo de derivados da soja proporciona.

Propostas para aumentar o consumo de derivados da soja, relatadas pelos consumidores, demonstraram que a opção 'mais investimentos em marketing' para divulgação destes alimentos foi citada por um número significativo de pessoas, seguida da necessidade de melhorar o sabor e fornecer mais informações à 
população sobre os benefícios do produto. Foi comentada também, em percentual elevado, a necessidade de ofertar maior variedade de produtos e proporcionar maior visibilidade destes nos pontos de venda.

Outras sugestões referiam-se à melhoria da textura dos alimentos, principalmente o leite de soja, que possui uma textura grossa; melhoria do aroma; diminuição dos preços; inovação, inserindo derivados da soja no preparo de alimentos nas refeições diárias; e incentivo ao público jovem, para haver uma mudança de hábito da futura população adulta, contribuindo para melhores índices na saúde da população.

\section{CONSIDERAÇÕES FINAIS}

Entre os diversos produtos do agronegócio brasileiro destaca-se a soja pelo papel relevante no Produto Interno Bruto e exportação. O Brasil é considerado com maior potencial de crescimento em termos de produção. A industrialização do produto, apesar de em menor proporção, também tem apresentado crescimento acentuado no país.

Contrastando com os índices positivos de produção, industrialização e da relevância da soja na alimentação humana, constatou-se que o consumo de alimentos derivados da soja ainda é incipiente no mercado consumidor brasileiro.

Os resultados da pesquisa demostraram que somente $34 \%$ afirmam consumir atualmente alimentos derivados da soja, 43\% já consumiram e deixaram de consumir e $23 \%$ relataram nunca ter consumido estes alimentos. Esse baixo consumo pode estar relacionado ao desconhecimento das vantagens relacionadas ao produto, fator também constatado pela pesquisa em que a maior parte da amostra de consumidores entrevistados relata que desconhece os benefícios da soja para a saúde (22\% dos entrevistados afirmam que desconhecem totalmente, enquanto $57 \%$ afirmam que possuem pouco conhecimento).

Para as indústrias alimentícias que estão no mercado produzindo derivados da soja é necessário investir nos aspectos sensoriais e na divulgação dos benefícios do consumo à saúde, instigando a compra. Para estimular o mercado consumidor as empresas podem aumentar a quantidade de informações através de campanhas na 
mídia, nos pontos de venda e nas próprias embalagens.

Desta forma, os resultados deste estudo apresentam informações que poderão ser utilizadas pelas empresas para obter maior representatividade no mercado e lucratividade e os consumidores terão mais conhecimento e oportunidade de consumir alimentos mais saudáveis.

\section{REFERÊNCIAS}

ADLERCREUTZ, H. Phyto-oestrogens and cancer. Lancet Oncol., v. 3, 2002.

AMARAL, V. M. G. A importância da soja como alimento funcional para qualidade de vida e saúde. 2006. 71f. Dissertação (Mestrado em Gestão da Qualidade Total) Universidade Estadual de Campinas, Campinas, 2006.

AMAZONAS, L. Conjuntura soja. Disponível em: < http://www.agricultura.gov. br/>. Acesso em: abr. 2013.

ANP. Agência Nacional de Petróleo, Gás Natural e Biocombustível. Anuário estatístico 2010. Disponível em: < http://www.anp.gov.br/?pg=31286>. Acesso em: abr. 2013.

ANVISA. Agência Nacional de Vigilância Sanitária. Alimentos. Disponível em: $<$ http://portal.anvisa.gov.br/wps/content/Anvisa + Portal/Anvisa/Inicio/Alimentos/Assuntos + de + Interesse/Alimentos + Com +Alegacoes + de + Propriedades + Funcionais $+\mathrm{e}+\mathrm{ou}+\mathrm{de}+$ Saude/Alegacoes $+\mathrm{de}+$ propriedade + funcional + aprovadas $>$. Acesso em: abr. 2013.

ABIOVE. Associação Brasileira de Indústria de Óleos Vegetais. Para entender a moratória da soja: o cultivo responsável. Disponível em: <http://www.abiove.org.br/ site $/$ index.php?page $=$ moratoria-dasoja $\&$ area $=$ NS0zLTE $=>$. Acesso em: dez. 2012.

BEHRENS, J. H.; SILVA, M. A. A. P. Atitude do consumidor em relação à soja e pro- 
dutos derivados. Ciência e Tecnologia de Alimentos, Campinas, v. 24, n. 3, p. 431-439, jul./set. 2004.

CARRÃO-PANIZZI, M. C.; MANDARINO, J. M. G. Soja: potencial de uso na dieta brasileira. Londrina: [s.n.], 1998.

CHANG, Y. K. Alimentos funcionais e aplicação tecnológica: padaria da saúde e centro de pesquisas em tecnologia de extrusão. In: SIMPÓSIO BRASILEIRO SOBRE OS BENEFÍCIOS DA SOJA PARA A SAÚDE HUMANA, 1., 2001, Londrina. Anais... Londrina: [s.n.], 2001. p. 41-45.

CHIARELLO, M. D. A soja e os alimentos funcionais: oportunidades de parcerias em P\&D para os setores público e privado. Revista Parcerias Estratégicas, Brasília, $n$. 15, p. 52-59, out. 2002.

CHURCHILL JR., G. A.; PETER J. P. Marketing: criando valor para os clientes. 2. ed. São Paulo: Saraiva, 2010.

COTRIJUC. Cooperativa Agropecuária Júlio de Castilhos. Disponível em: < http:// www.cotrijuc.com.br/site/cotrijuc.php?mod=historico > . Acesso em: maio 2015.

DEMARCHI, M. Soja. Curitiba: Departamento de Economia Rural, 2011.

EMBRAPA. Tecnologias de produção de soja região central do Brasil 2004. Disponível em: <http://www.cnpso.embrapa.br/producaosoja/SojanoBrasil.htm>. Acesso em: abr. 2013.

FONTANA, D. C. et al. Modelo de estimativa de rendimento de soja no Estado do Rio Grande do Sul. Pesquisa Agropecuária Brasileira, Brasília, v. 36, n. 3, p. 399-403, mar. 2001.

FREITAS, M. C. M. A cultura da soja no Brasil: o crescimento da produção brasileira e o surgimento de uma nova fronteira agrícola. Enciclopédia Biosfera, Goiânia, v. 7, n. 12, p. 1-12, maio 2011. 
GIGLIO, E. M. O comportamento do consumidor. São Paulo: Thomson, 2010.

IBGE. Instituto Brasileiro de Geografia e estatística. Disponível em: < http://www.cidades.ibge.gov.br/xtras/perfil.php?codmun $=431120 \&$ search $=$ rio-grande-do-sul $\mid$ julio-de-castilhos\&lang= > . Acesso em: maio 2015.

IBGE. Levantamento sistemático da produção agrícola. Disponível em: <http:// www.ibge.gov.br/home/estatistica/indicadores/agropecuaria/lspa/spa_201207. pdf> . Acesso em: nov. 2012.

KINOUCHI, F. L. Iogurte de soja como coadjuvante no tratamento de câncer de mama. 2006. 93f. Tese (Doutorado em Análises Clínicas) - UNESP, São Paulo, 2006.

KOTLER, P.; KELLER, K. L. Administração de marketing. 12. ed. São Paulo: Pearson Prentice Hall, 2006.

LAZZAROTTO, J. J.; HIRAKURI, M. H. Evolução e perspectivas de desempenho. Londrina: Embrapa Soja, 2010.

MALHOTRA, N. K. Pesquisa de marketing: uma orientação aplicada. 4. ed. Porto Alegre: Bookman, 2006.

MANDARINO, J. M. G. A soja e a saúde humana. In: ENCONTRO FRANCO-BRASILEIRO DE BIOCIÊNCIA E BIOTECNOLOGIA: ALIMENTOS FUNCIONAIS E NUTRACÊUTICOS, 2002, Brasília. Resumos das Palestras... Brasília: [s.n.], 2002. p. 9-11.

MAPA. Ministério da Agricultura, Pecuária e Abastecimento. Câmara setorial da soja. Disponível em: <http://www.agricultura.gov.br/vegetal/culturas/soja/saiba -mais > . Acesso em: abr. 2013.

MESSINA, M. J. et al. Soy intake and câncer risk: rewiew of the in vitro and in vivo data. Nutr. Cancer, v. 2, 1994.

MOREIRA, M. G. Soja: análise da conjuntura agropecuária. Disponível em: <http:// 
www.agricultura.pr.gov.br>. Acesso em: jul. 2013.

PENHA, L. A. O. et al. A soja como alimento: valor nutricional, benefícios para a saúde e cultivo orgânico. B.CEPPA, Curitiba, v. 25, n. 1, p. 91-102, jan./jun. 2007.

SANTOS, A. B.; BACHA, C. J. C. A evolução da cultura e do processamento industrial da soja no Brasil - período de 1970 a 2002. Teoria e Evidência Econômica, Passo Fundo, v. 11, n. 20, p. 89-118, mai. 2003.

SANTOS, C. et al. Anuário brasileiro da soja 2011. Santa Cruz: Gazeta, 2011.

SCHIFFMANN, L. G. Comportamento do consumidor. Rio de Janeiro: LTC, 2009.

SCHLESINGER, S. Soja: o grão que segue crescendo. Grupo de Trabalho sobre desenvolvimento e meio ambiente nas Américas. 2008.

SOLOMON, M. R. Comportamento do consumidor: comprando, possuindo e sendo. Porto Alegre: Bookman, 2011.

VENCATO, A. Z. et al. Anuário brasileiro da soja 2010. Santa Cruz: Gazeta, 2010.

YIM, D. K. Soja: um arsenal milenar aliado à saúde. In: ENCONTRO FRANCO-BRASILEIRO DE BIOCIÊNCIA E BIOTECNOLOGIA - ALIMENTOS FUNCIONAIS E NUTRACÊUTICOS, Anais.... Brasília: Embrapa, 2002. p. 12. (Embrapa. Documentos, 85). Disponível em: < http://www.cenargen.embrapa.br/publica/trabalhos/doc085. pdf $>$. Acesso em: jan. 2013.

Recebido em: 16 de maio de 2014 Aceito em: 09 de junbo de 2015 